Lavigne Delville, Philippe, Toulmin, Camilla \& Traoré, Samba (dir.). - Gérer le foncier rural en Afrique de l'Ouest. Dynamiques foncières et interventions publiques. Paris, Karthala ; Saint-Louis (Sénégal), URED, 2000, 357 p.

Jean-Pierre Jacob

\title{
OpenEdition
}

Journals

Édition électronique

URL : https://journals.openedition.org/etudesafricaines/1490

DOI : 10.4000/etudesafricaines. 1490

ISSN : $1777-5353$

Éditeur

Éditions de l'EHESS

Édition imprimée

Date de publication : 1 janvier 2002

ISBN : 978-2-7132-1429-5

ISSN : 0008-0055

Référence électronique

Jean-Pierre Jacob, « Lavigne Delville, Philippe, Toulmin, Camilla \& Traoré, Samba (dir.). - Gérer le foncier rural en Afrique de l'Ouest. Dynamiques foncières et interventions publiques. Paris, Karthala ; SaintLouis (Sénégal), URED, 2000, 357 p. », Cahiers d'études africaines [En ligne], 166 | 2002, mis en ligne le 10 juin 2005, consulté le 16 juin 2021. URL : http://journals.openedition.org/etudesafricaines/1490 DOI : https://doi.org/10.4000/etudesafricaines.1490

Ce document a été généré automatiquement le 16 juin 2021.

(c) Cahiers d'Études africaines 


\section{Lavigne Delville, Philippe, Toulmin, Camilla \& Traoré, Samba (dir.). - Gérer le foncier rural en Afrique de l'Ouest. Dynamiques foncières et interventions publiques. Paris, Karthala ; Saint-Louis (Sénégal), URED, 2000, 357 p.}

Jean-Pierre Jacob

1 Les «éditeurs » de Gérer le foncier rural en Afrique de l'Ouest proposent un ouvrage qui inclut des études de cas concernant l'Afrique anglophone (Ghana avec Kasanga, Amanor, Abudulai et Bortei-Doku Aryeetey, Cameroun avec Mope Simo et Egbe) et, pour les pays d'Afrique francophone plus connus (Côte-d'Ivoire, Burkina Faso, Niger, Mali, Sénégal, Bénin), des présentations d'expériences en cours visant l'amélioration des systèmes de gestion locale des ressources. L'ensemble du recueil témoigne en outre d'un intérêt certain pour les droits des « minorités » dans les processus de changement actuel (les éleveurs avec Traoré et Sanou, les femmes avec Bortei-Doku Aryeetey) et pour les questions de gestion de ressources spécifiques : la forêt (Kesse, Egbe, Amanor) et l'eau (Kassibo). Ce faisant, il est proposé au lecteur un volume de bonne tenue, varié dans les thèmes qu'il aborde et les terrains qu'il présente, et presque intégralement constitué de contributions de chercheurs du Sud, parmi les meilleurs spécialistes du sujet.

2 Une première réflexion est inspirée de la structure même de l'ouvrage, articulé grosso modo, comme je l'évoquais précédemment, autour de l'axe problèmes/solutions. La première partie de l'ouvrage est composée d'analyses sociopolitiques de la question foncière, tandis que les deux autres sont consacrées à la présentation d'innovations 
institutionnelles censées faciliter la régulation entre acteurs, tributaires des mêmes ressources pour des usages alternatifs ou concurrents. Cette structure ne présente pas que des avantages, dans la mesure où les terrains sur lesquels ont porté les analyses (Ghana, Cameroun) ne correspondent pratiquement pas aux terrains de l'action (Afrique francophone). Le livre est cependant suffisamment riche pour que le thème du lien entre connaissance et action puisse être reconstruit de manière transversale, en dépassant les obstacles cognitifs évidents que peut provoquer ici la séparation géographique des deux démarches. Pour explorer ce lien, il serait possible de reprendre, par exemple, l'hypothèse forte $d u$ "pluralisme institutionnel " avancée dans l'introduction et d'examiner le sort qui lui est réservée dans les dispositifs opérationnels présentés par certains contributeurs (Kesse, Hounkpodote ou Yacouba) : les outils proposés constituent-ils des réponses satisfaisantes à ce pluralisme? Qu'estce qui fait la différence entre une «bonne » innovation institutionnelle, apte à réguler « les jeux d'acteurs complexes, jouant sur la pluralité des normes, au sein de systèmes fonciers locaux hybrides» (Lavigne Delville, Toulmin \& Traoré, p. 23), et une «mauvaise» innovation, capable seulement d'ajouter aux ressources du répertoire stratégique des individus?

3 Une autre question-clé court, en filigrane, dans la quasi-totalité du recueil qui nous est présenté : celle de savoir au nom de quelle théorie de la justice il convient d'agir en matière foncière. Une première approche de cette question - nous permettant d'avoir un aperçu sur ce que pourrait être une théorie «émique » du problème - est proposée grâce aux études de cas de la première partie. Ce qui frappe en effet à leur lecture est l'état d'impuissance des systèmes politiques ghanéen et camerounais, étant donné la marchandisation des ressources naturelles, à assurer plus longtemps le respect des libertés et des capacités des petits producteurs, au sens d'Amartya Sen ${ }^{1}$ (1999) de "capabilities», "droits humains aux capacités de base». Ces petits producteurs, "propriétaires » traditionnels des ressources progressivement dépossédés par une certaine institutionnalisation de la " coutume » dès l'époque coloniale (une «tradition inventée » comme le dit Amanor, p. 103 ; voir également sur le sujet la présentation très complète de Kasanga), ne sont plus en position, du fait de leur perte de maitrise sur leur environnement, de satisfaire ni leurs droits à la « bonne vie », ni $a$ fortiori celles des autres hommes (leurs contemporains demandeurs de terres, mais aussi et surtout, bien entendu, leurs enfants). L'importance des investissements capitalistiques dans le domaine des ressources naturelles et la lutte constante pour les moyens d'existence qu'ils provoquent (Amanor, p. 121), rend impossible le maintien chez les agriculteurs d'une vision du foncier comme lien symbolique, leur permettant de faire la jonction avec leurs prédécesseurs (les ancêtres), avec leurs contemporains (devenus des concurrents) et avec leurs successeurs (les générations à venir). La remarque des femmes de la périphérie de Tamale rapportée par S. Abudulai est, de ce point de vue, tout à fait significative : « Si, pauvres comme nous sommes, nous ne pouvons acquérir des terrains aux prix actuels, qu'en sera-t-il de nos enfants?» (p. 159).

Ce qui est exprimé au Ghana sur le mode du désespoir, peut être éclairé par des études anthropologiques sur d'autres sociétés ouest-africaines, dans lesquelles ces représentations sont évoquées probablement d'autant plus complètement et librement que la compétition pour les ressources entre communautés et investisseurs privés y est plus faible. J'ai entrepris pour ma part des enquêtes intensives sur les raisons qu'ont les autochtones winye du centre-ouest du Burkina Faso, qui ont pourtant une certaine expérience de la monétarisation de l'économie au travers de la production cotonnière, 
de refuser de vendre la terre. Les arguments qu'ils proposent en réponse à mes questions sont variés, mais je pense qu'on peut les synthétiser assez fidèlement en disant qu'ils sont proches d'un refus d'adopter le « paradoxe de la démocratie » tel qu'il a été défini par John Elster: "Chaque génération veut être libre d'imposer des contraintes aux générations suivantes, tout en refusant les contraintes imposées par les générations précédentes ${ }^{2}$. D'une part, les autochtones ne veulent pas vendre (ou prêter sans conditions) leurs terres parce qu'ils refusent de mettre en danger la base sur laquelle ils peuvent mener une vie de producteurs libres. D'autre part - et c'est là qu'entre en jeu leur conception de la justice "transgénérationnelle »- ils cherchent à savoir, avant de répondre aux demandes de terres de leurs contemporains, si, ce faisant, ils ne mettent pas en danger le droit des générations futures à jouir de cette possibilité qu'ils ont eu, grâce à leurs ancêtres, de rester « maîtres de leur monde » ${ }^{3}$. Ils ne sont donc guère sensibles aux appels à la « démocratisation » de l'accès au foncier par le marché ou par les recommandations de l'État ou des intervenants externes, parce qu'ils cherchent à reconduire ces liens entre identité et espace dans la durée ${ }^{4}$. C'est, me semble-t-il, la même volonté de sécurisation foncière, appréhendée dans la diachronie, au nom du droit à la pérennisation d'un certain mode de vie, qui anime les groupes ethnoprofessionnels (éleveurs et pêcheurs) décrits respectivement par Samba Traoré, Saïdou Sanou et Bréhima Kassibo. Mais il faut dire que l'opinion informée commence à admettre que ces groupes - et notamment les éleveurs - ont été généralement très maltraités par les différentes politiques agricoles des États ouest-africains, ces discriminations leur conférant une sorte de droit moral à récupérer leur " historicité ». Ils peuvent à présent avancer avec une certaine légitimité que la sécurisation et la pérennisation de l'exploitation des zones qui leur sont traditionnellement réservées (voir notamment Sanou, p. 279) est une bonne chose, parce que, si cet objectif n'est pas respecté, ils risquent de disparaître à tout jamais du paysage sahélien. Ce combat symbolique est évidemment beaucoup plus difficile à mener par un groupe d'agriculteurs autochtones.

5 L'introduction de l'ouvrage défend une autre conception de la justice, fondée sur l'égalité d'opportunité (accès égal), notamment pour la terre ou les ressources forestières. Les éditeurs soulignent en effet qu'au niveau pratique, il ne sert à rien d'admettre le principe d'une gestion "plus locale» des ressources, si cette dévolution n'est pas faite dans une logique d'inclusion de tous les usagers (p. 32 et p. 44). Cette démarche est illustrée par la description de la mise en place d'une série d'instruments participatifs (commissions paysans-forêts en Côte-d'Ivoire par Kesse, plan foncier rural au Bénin par Hounkpodote, commissions foncières au Niger par Yacouba) permettant de recenser, d'enregistrer et de sécuriser l'ensemble des droits (droits d'appropriation et droits d'usage) sur la base d'une situation foncière telle qu'elle se présente à l'instant $t$. Les forces et les faiblesses de la démarche dont s'inspirent ces outils sont évidemment inverses de celles qu'impliquent la première conception "émique» de la justice. Privilégiant une optique de justice distributive dans la synchronie, cette démarche compte sur les négociations futures pour lever les contraintes que les engagements présents sur les pratiques à venir ne manqueront pas de poser ${ }^{5}$. En fin de compte, pour être efficaces, les innovations proposées doivent réunir deux conditions: que les autochtones oublient la conception de justice "transgénérationnelle " qu'ils ont entretenue jusqu'au moment de l'intervention; et que les générations autochtones qui vont se succéder à partir du moment où est pris l'engagement contractuel n'oublient pas la conception de justice distributive sur laquelle est fondé cet engagement, et qu'ils 
ne se mettent pas à agir de manière «moderne », par exemple en se conformant au paradoxe de la démocratie d'Elster.

6 Autrement dit, il est demandé aux autochtones d'oublier leur temporalité propre et d'adopter, avec leurs contemporains allochtones, à la suite d'une véritable opération "d'enculturation», une autre temporalité, fondement d'une communauté d'appartenance nouvelle, composée par l'ensemble des parties prenantes vivant des ressources naturelles dans un espace et à un moment donné ${ }^{6}$. Je crains, malheureusement, que ces conditions soient très difficiles à remplir, comme le montre l'observation dans la durée du sort des arrangements informels permettant à des usagers d'avoir accès à la terre, ces arrangements étant assez systématiquement défaits par les fils des prêteurs de terres une fois leurs pères disparus ${ }^{7}$. Ce n'est évidemment pas un mince mérite de l'ouvrage dirigé par P. Lavigne Delville, C. Toulmin et S. Traoré que d'avoir contribué à lancer ce débat relativement nouveau sur les conditions spatiotemporelles de l'appropriation des politiques foncières, dans un contexte qui reste largement dominé par les approches « techniques».

\section{NOTES}

1. SEN, A., Development as Freedom, New York, A. Knopf, 1999.

2. ELSTER, John, Le laboureur et ses enfants. Deux essais sur les limites de la rationalité, Paris, Éditions de Minuit, 1986 : 169.

3. La plupart des Winye ayant fait des séjours prolongés en Côte-d'Ivoire, ils ont assisté au dessaisissement foncier des autochtones de l'Ouest forestier ivoirien.

4. Je ne crois pas que ces représentations soient très anciennes. L'attitude qui prévalait auparavant dans la zone, longtemps considérée comme sous-peuplée, était d'accueillir sans réserve les nouveaux arrivants. Cela permettait aux tuteurs " d'avoir des fesses ", de réunir du monde derrière soi, ce qui est toujours utile lorsqu'on veut jouer un rôle économique ou politique important dans une communauté. J'aurais tendance à dater ces « nouvelles » conceptions de la période post-indépendance (1960), car c'est à cette époque que se font jour les premières migrations mossi massives vers l'Ouest (liées au coton) et qu'apparaissent également les premiers politiciens autochtones qui - parfois contre les consignes de leur parti - attirent l'attention de leurs « frères » sur le danger qu'il y a à distribuer trop de terre aux " étrangers ».

5. En fait le seul outil qui semble relever pleinement ce défi est le plan foncier rural (PFR) décrit par Hounkpodote, qui propose un véritable espace/temps de réglement des questions foncières, alternatif de l'espace/temps selon lequel les sociétés autochtones traitent de ces questions (voir, pour la dimension spatiale, la manière dont le PFR privilégie le niveau de décision villageois, p. 233), alors que nombre de tutorats fonciers « traditionnels » impliquent des relations supra villageoises. L'incapacité des autres outils (voir Kesse, p. 218 ; Yacouba, p. 240) à prendre en compte les conflits actuels sur les ressources laisse assez mal augurer de leurs capacités régulatrices, a fortiori de leurs possibilités à faire sens dans le futur. 
6. Sur la question plus générale du développement comme essai de constitution d'un espace-temps nouveau, voir Jean-Pierre Jacob, « Connaissance et développement en Afrique », in J.-P. ЈАСОВ (dir.), Sciences sociales et coopération en Afrique : les rendez-vous manqués, 2000 : 11-30 (Nouveaux Cahiers de l'IUED, nº 10).

7. Pour l'Ouest du Burkina voir Mahamadou Zongo \& Paul MATHIEU, « Transactions foncières marchandes dans l'ouest du Burkina Faso : vulnérabilité, conflits, sécurisation, insécurisation ", Communication pour le Colloque de l'APAD, Saint-Louis, 26-28 janvier 2000, ronéo, 2000. De la même manière, dans des contextes de forte marchandisation de la terre comme en Côte-d'Ivoire, il arrive fréquemment que les fils ne reconnaissent pas les ventes de fonds réalisés par leurs pères, une fois ceux-ci décédés. 